\title{
Optimal balanced measurement designs when errors are correlated
}

\author{
C.T. Liao ${ }^{\mathrm{a}}$, C.H. Taylor ${ }^{\mathrm{b}}$, H.K. Iyer ${ }^{\mathrm{c}, *}$ \\ ${ }^{a}$ Department of Agronomy, National Taiwan University, Taipei, Taiwan \\ ${ }^{\mathrm{b}}$ Proctor and Gamble, Cincinnatti, OH, USA \\ ${ }^{\mathrm{c}}$ Department of Statistics, Colorado State University, Ft. Collins, CO 80523, USA
}

Received 1 October 1995; received in revised form 10 April 1998; accepted 8 May 1999

\begin{abstract}
We consider situations in which some characteristic is to be measured on each of several specimens. For instance, the characteristic may be the concentration of lead in soil samples and a laboratory may routinely analyze samples from different customers. A measuring device is used to obtain the responses and the measurement errors generally exhibit serial correlation and/or drift. To help adjust the instrument readings for this serial correlation, standards are interspersed among the specimens at appropriate intervals. In this paper we present the results of an investigation of balanced measurement designs that minimize the uncertainty in the adjusted measurements under an $\mathrm{AR}(1)$ error structure. Examples are given to illustrate the results. (C) 2000 Elsevier Science B.V. All rights reserved.
\end{abstract}

Keywords: Calibration problems; AR(1) process; Systematic errors; Random errors; BLUE; A-optimality

\section{Introduction}

A measurement process is frequently subject to errors which are generally classified as systematic, or random, or a combination of both. The random errors are defined to have a zero expected value, and the systematic errors are defined to be due to biases in the measurement process. Thus, the measured (observed) value of an unknown specimen can be described by the following additive model:

$$
y_{i}=\mu_{i}+\tau_{i}+\varepsilon_{i}
$$

where $\mu_{i}, \tau_{i}$ and $\varepsilon_{i}$ denote the systematic error, the true value, and the random error, respectively, corresponding to the specimen being measured. If the errors can be estimated sufficiently precisely, then it may be possible to process the raw measurement

\footnotetext{
* Corresponding author. Tel.: +1-970-491-6870; fax: +1-970-491-7895.

E-mail address: hari@stat.colostate.edu (H.K. Iyer)
} 
to produce an estimate of the true value which is "better" than the raw observation itself.

Since "standards" have known true values, the errors associated with the measurement process are "observed" whenever a standard is measured. Typically, random errors are assumed to be independent, but it is often more realistic to acknowledge that the measurement process is serially correlated. For example, consider an automated system which measures the concentrations of a toxic material in water samples contained in each of several test tubes. These measurements are made sequentially in time. If the time period between these measurements is relatively small, then it is quite conceivable that a substantial correlation may exist between consecutive random errors. Suppose the correlation structure can be modeled sufficiently well so that it may be assumed known. Then a measurement experiment consisting of several observations of each of $m$ different unknown specimens and of a known standard may utilize the "known" correlation structure to give better estimates of the unknowns. The following example is used to illustrate how this occurs.

Example 1.1. Consider the simple situation where there are only random errors, i.e., the systematic errors are assumed to be zero. Let $y_{1}$ represent an observation of a standard and $y_{2}$ represent an observation of an unknown, and $\tau_{1}$ and $\tau_{2}$ represent the true values of the standard and the unknown, respectively. The relationship between the observed values and the true values may be written as

$$
\left[\begin{array}{l}
y_{1} \\
y_{2}
\end{array}\right]=\left[\begin{array}{l}
\tau_{1} \\
\tau_{2}
\end{array}\right]+\left[\begin{array}{l}
\varepsilon_{1} \\
\varepsilon_{2}
\end{array}\right] .
$$

Also suppose the random errors have the distribution

$$
\left[\begin{array}{l}
\varepsilon_{1} \\
\varepsilon_{2}
\end{array}\right] \sim N\left(\left[\begin{array}{l}
0 \\
0
\end{array}\right],\left[\begin{array}{ll}
1.0 & 0.9 \\
0.9 & 1.0
\end{array}\right]\right) .
$$

Since $\varepsilon_{1}$ is observable, $\varepsilon_{2}$ may be estimated by

$$
E\left(\varepsilon_{2} \mid \varepsilon_{1}\right)=0.9 \varepsilon_{1} \text {. }
$$

Consequently, the unknown quantity may be estimated as

$$
\hat{\tau}_{2}=y_{2}-0.9 \varepsilon_{1} \text {. }
$$

The variance of this estimate is

$$
\operatorname{Var}\left(\hat{\tau}_{2}\right)=0.19
$$

which is less than 1 , the variance of $y_{2}$.

The precision in estimating the true values of unknown specimens also depends on the arrangement (ordering) of the observations of the unknowns and the standard. The following example illustrates this phenomenon.

Example 1.2. Suppose a single measurement of one unknown is to be made along with two observations of the standard. The systematic errors are assumed to be zero, 
and the random errors of observations 1,2 , and 3 are normally distributed with mean zero and the covariance matrix

$$
\left[\begin{array}{lll}
1.00 & 0.50 & 0.25 \\
0.50 & 1.00 & 0.50 \\
0.25 & 0.50 & 1.00
\end{array}\right] \text {. }
$$

If the observations are made in the order USS where $U$ denotes the unknown specimen and $S$ denotes the standard, then the unknown quantity may be estimated as

$$
\hat{\tau}_{1}=y_{1}-E\left(\varepsilon_{1} \mid \varepsilon_{2}, \varepsilon_{3}\right)=y_{1}-0.5 \varepsilon_{2} .
$$

The variance of this estimate is

$$
\operatorname{Var}\left(\hat{\tau}_{1}\right)=0.75
$$

If the observations are made in the order $S U S$, then the unknown quantity may be estimated as

$$
\hat{\tau}_{2}=y_{2}-E\left(\varepsilon_{2} \mid \varepsilon_{1}, \varepsilon_{3}\right)=y_{2}-0.4 \varepsilon_{1}-0.4 \varepsilon_{3} .
$$

The variance of this estimate is

$$
\operatorname{Var}\left(\hat{\tau}_{2}\right)=0.60
$$

So the order SUS is preferable to the order USS.

Literature pertaining to calibration or measurement problems involving correlated errors appears to begin with Pepper (1973). He considered the following model for the measurement process:

$$
y_{t}=\tau_{t}+\left(b_{t}+\eta_{t}\right)
$$

where $y_{t}$ represents the measured value and $\tau_{t}$ represents the corresponding true value, for $t=1,2, \ldots, n$. The quantity $\left(b_{t}+\eta_{t}\right)$ represents random errors, where $\eta_{t}$ are independent $N\left(0, \sigma_{\eta}^{2}\right)$ random variables, and $b_{t}$ arise from a random walk of the form

$$
b_{t}=b_{t-1}+\varepsilon_{t}
$$

with $\varepsilon_{t}$ independent $N\left(0, \sigma_{\varepsilon}^{2}\right)$ random varibles which are also independent of $\eta_{t}$. Pepper (1973) provided three different measurement policies. For each of the policies, he gave approximate maximum likelihood estimators for the unknowns. In addition, he gave suggestions for ordering the observations based on enumeration of all possible designs in situations where the total number of observations is "small".

By a suitable reparameterization, it is easy to see that the problem addressed in this paper is equivalent to the problem of comparing a set of treatments with a control using an unblocked experimental design, that is, using only one block of size equal to the total number of runs. Considerable attention has been devoted in the literature to the problem of comparing treatments with a control, but nearly all of the work is under the assumption of uncorrelated errors. Cutler (1993) does consider this problem under a circular AR(1) error structure as well as a linear AR(1) error structure and demonstrates the A-optimality of certain classes of binary block designs. Other authors have discussed 
block designs with an $\mathrm{AR}(1)$ error structure but they concern themselves with the estimation of all pairwise treatment differences rather than comparing treatments with a control. See, for instance, Berenblut and Webb (1974), Cheng (1983, 1988), Ipinyomi (1986), Kiefer (1960), Kiefer and Wynn (1981, 1983, 1984), Kunert (1985a,b, 1987), Martin (1982), Martin and Eccleston (1991), Russell and Eccleston (1987a, b), and Williams (1949, 1985, 1986). Another useful source of information on this topic is the review article by Hedayat et al. (1988). Optimal designs for the comparision of treatments to a control using a model with uncorrelated errors are treated by Hedayat et al. (1988), Pigeon and Raghavarao (1987) and Majumdar (1988). None of these articles directly address the problem under consideration here. Although Cutler (1993) considers the same error structure as we do, his focus is on binary block designs whereas we use single block designs that are nonbinary.

In the next section we describe our problem and point out its equivalence with the problem of comparing all treatments to a control. Section 3 provides optimal designs, within the class of "balanced" designs, when there are three or more unknowns to be measured. For situations where at most two unknowns are to be measured we can actually find optimal designs in the class of all designs but we do not present the results here. Instead the reader is referred to Taylor (1989). Section 4 presents an algorithm to generate the optimal balanced designs. Proofs of the theroems in Section 3 are given in the appendix.

\section{Preliminaries}

\subsection{The problem}

The specific problem treated in this paper assumes that a total of $N$ measurements can be made some of which may be measurements on a standard while the remaining measurements are of $m$ different unknown specimens. The standard as well as each of the specimens may be measured more than once if so desired. The number of measurements of unknown specimen $i$ is denoted by $t_{i}, i=1,2, \ldots, m$, and the number of measurements of the standard is denoted by $b-1(b>1)$. Furthermore these observations are made sequentially in time. The statistical model assumes a constant but unknown systematic error, say $\mu$. Let $\tau_{0}$ be the true value of the standard (which is, of course, known), and $\delta_{i j}$ be the indicator function defined by

$$
\delta_{i j}=\left\{\begin{array}{l}
1 \text { if } j=0 \text { and observation } i \text { is of the standard, } \\
1 \text { if } j \in\{1,2, \ldots, m\} \text { and observation } i \text { is of unknown } j \\
0 \text { otherwise. }
\end{array}\right.
$$

Then, the model described in (1.1) can be rewritten as

$$
y_{i}=\mu+\delta_{i 0} \tau_{0}+\sum_{j=1}^{m} \delta_{i j} \tau_{j}+\varepsilon_{i} \quad i=1, \ldots, N .
$$


The random errors $\varepsilon_{i}$ are now assumed to arise from a first-order stationary autoregressive process $(\mathrm{AR}(1))$ of the form

$$
\varepsilon_{i}=\phi \varepsilon_{i-1}+z_{i}
$$

where $z_{i}$ are assumed to be uncorrelated random variables with zero means and equal variances $\sigma^{2}$. The coefficient $\phi(-1<\phi<1)$ is assumed known. We use a stationary $A R(1)$ error sequence rather than a finite $A R(1)$ error sequence because of the inverse of the covariance matrix has a rather simple form in the former case. We are interested in estimating $\tau_{1}, \ldots, \tau_{m}$ most efficiently.

Let us reparameterize the model as follows. Define $\theta_{0}=\mu$ and $\theta_{i}=\mu+\tau_{i}$ for $i=1, \ldots, m$. Also let $z_{i}=y_{i}-\delta_{i 0} \tau_{0}$. The model equation in (2.1) becomes

$$
z_{i}=\sum_{j=0}^{m} \delta_{i j} \theta_{j}+\varepsilon_{i}, \quad i=1, \ldots, N
$$

which is a one-way classification model with $m+1$ treatments with means $\theta_{0}, \ldots, \theta_{m}$. The parameter $\theta_{0}$ is for the control and the differences $\theta_{j}-\theta_{0}$ are precisely the $\tau_{j}$ 's for $j=1, \ldots, m$.

This paper is concerned with finding optimum designs for estimating the values of the unknown specimens within the class of "balanced" measurement designs (to be defined shortly). In particular, we consider the A-optimality criterion according to which the average variance of the best linear unbiased estimates (BLUE) of the true values of the unknown specimens is minimized. The following terminology is relevant to the rest of the paper.

\subsection{The class of "balanced measurement designs"}

A balanced measurement design is defined here as a design for which the BLUEs of the true values of the unknown specimens have the same variance and all pairs of the estimators have the same covariance. Such a design is often desirable since inferences may be made without regard to the labelling of the specimens.

\subsubsection{Batches}

Designs used in this paper will be described in terms of "batches". A "batch" is defined to be the set of all measurements of unknown specimens between two successive observations of the standard, or the set of all measurements (if any) of unknowns before the first measurement of a standard, or the set of all measurements (if any) of unknown specimens after the last measurement of a standard. A batch of measurements is called an "interior batch" if the corresponding measurements are sandwiched between measurements of the standard. If the batch of measurements forms the initial segment of the measurement process it is called the "initial batch" and if it forms the final segment of the measurement experiment, then it is called the "final batch".

Empty batches are permissible and these occur when the standard is measured at two consecutive time points with no unknown in between. The number of empty batches is 
denoted by $b_{0}$. As a consequence of these definitions, $b-1$ observations of a standard give rise to $b$ batches. The following example is given to illustrate the above definitions.

Example 2.1. Consider the following sequence of the observations.

$$
U_{1} U_{3} S U_{1} U_{3} U_{3} U_{3} U_{2} U_{1} U_{2} U_{2} S S
$$

where $U_{i}$ denotes the unknown specimen $i$ and $S$ denotes the standard. In this case, there are $b=4$ batches. Of these 4 batches $b_{0}=2$ are empty. The initial batch consists of two measurements, the first being of specimen 1 and the next of specimen 3 . The first interior batch consists of the eight measurements in the order $U_{1} U_{3} U_{3} U_{3} U_{2} U_{1} U_{2} U_{2}$. The second interior batch is empty since we have two successive measurements of the standard with no unknown specimen in between. The final batch is also empty since no unknowns are measured after the last measurement of the standard. Note that specimen 1 is measured $t_{1}=3$ times, specimen 2 is measured $t_{2}=3$ times, and specimen 3 is measured $t_{3}=4$ times. The total number of measurements is $N=(b-1)+t_{1}+t_{2}+t_{3}=13$.

\subsection{BLUEs for the unknown true values}

Since $\tau_{0}$ is known in the model described in (2.1), we let

$$
z_{i}= \begin{cases}y_{i} & \text { if observation } i \text { is of the unknown } \\ y_{i}-\tau_{0} & \text { if observation } i \text { is of the standard }\end{cases}
$$

Thus, we may write

$$
z_{i}=\mu+\sum_{j=1}^{m} \delta_{i j} \tau_{j}+\varepsilon_{i}
$$

or in matrix notation, this can be represented as

$$
\boldsymbol{z}=\boldsymbol{X} \boldsymbol{\beta}+\boldsymbol{\epsilon}
$$

where $\boldsymbol{X}$ is $n \times(1+m)$ defined as

$$
X_{i, j}= \begin{cases}1 & \text { if } j=1, \\ \delta_{i, j} & \text { if } j>1,\end{cases}
$$

and $\boldsymbol{\beta}=[\mu, \tau]^{\prime}$ where $\tau=\left[\tau_{1}, \tau_{2}, \ldots, \tau_{m}\right]$. We write $\boldsymbol{X}=\left[\boldsymbol{X}_{1}, \boldsymbol{X}_{2}\right]$ where $\boldsymbol{X}_{1}$ is a column of 1 's and $\boldsymbol{X}_{2}$ has $\delta_{i, j}$ as its $(i, j)$-element.

The random errors $\varepsilon_{i}$ are assumed to arise from an $\mathrm{AR}(1)$ process described in (2.2). Hence, the vector of these random errors $\epsilon=\left[\varepsilon_{1}, \varepsilon_{2}, \ldots, \varepsilon_{N}\right]^{\prime}$ has a distribution with a zero mean and a variance-covariance matrix $\sigma^{2} \boldsymbol{V}$, where $\boldsymbol{V}$ is a positive definite matrix with the elements

$$
V_{i, j}=\frac{\phi^{|i-j|}}{1-\phi^{2}}
$$


It is well known (see Siddiqui (1958)) that $\boldsymbol{V}^{-1}$ is given by

$$
\left(V^{-1}\right)_{i, j}= \begin{cases}1 & \text { if } i=j=1 \text { or } i=j=N \\ 1+\phi^{2} & \text { if } i=j \text { and } 1<j<N \\ -\phi & \text { if }|i-j|=1 \\ 0 & \text { otherwise. }\end{cases}
$$

The BLUE of $\boldsymbol{\beta}$ is

$$
\hat{\boldsymbol{\beta}}=[\hat{\mu}, \hat{\tau}]^{\prime}=\left(\boldsymbol{X}^{\prime} \boldsymbol{V}^{-1} \boldsymbol{X}\right)^{-1} \boldsymbol{X}^{\prime} \boldsymbol{V}^{-1} \boldsymbol{z}
$$

and its variance is

$$
\operatorname{Var}(\hat{\boldsymbol{\beta}})=\sigma^{2}\left(\boldsymbol{X}^{\prime} \boldsymbol{V}^{-1} \boldsymbol{X}\right)^{-1}
$$

From this it is easy to see that the BLUE of $\tau$ is given by

$$
\hat{\tau}=\boldsymbol{C}^{-1} \boldsymbol{X}_{2}^{\prime}\left[\boldsymbol{V}^{-1}-\boldsymbol{V}^{-1} \boldsymbol{X}_{1}\left(\boldsymbol{X}_{1}^{\prime} \boldsymbol{V}^{-1} \boldsymbol{X}_{1}\right)^{-1} \boldsymbol{X}_{1}^{\prime} \boldsymbol{V}^{-1}\right] \boldsymbol{z}
$$

and that

$$
\operatorname{Var}(\hat{\tau})=\sigma^{2} C^{-1}
$$

where

$$
\boldsymbol{C}=\boldsymbol{X}_{2}^{\prime}\left[\boldsymbol{V}^{-1}-\boldsymbol{V}^{-1} \boldsymbol{X}_{1}\left(\boldsymbol{X}_{1}^{\prime} \boldsymbol{V}^{-1} \boldsymbol{X}_{1}\right)^{-1} \boldsymbol{X}_{1}^{\prime} \boldsymbol{V}^{-1}\right] \boldsymbol{X}_{2} .
$$

The matrix $\boldsymbol{C}$ will be called the information matrix for $\tau$.

At this point it is useful to recast the measurement design of Example 2.1 in terms of treatments and controls and point the similarities and differences between the problem considered in this paper and the one addressed by Cutler (1993). Example 2.2 serves this purpose.

Example 2.2. Consider the design

$$
U_{1} U_{3} U_{0} U_{1} U_{3} U_{3} U_{3} U_{2} U_{1} U_{2} U_{2} U_{0} U_{0}
$$

which is obtained by replacing $S$ with $U_{0}$ in the measurement sequence of Example 2.1. Here $U_{0}$ represents the control and $U_{1}, U_{2}, U_{3}$ represent the treatments. If we are interested in comparing each treatment with the control, then, by virtue of the equivalence between measurement designs and treatment-control designs, the information matrix for $\left(\tau_{1}, \tau_{2}, \tau_{3}\right)$ given in (2.6) is identical to that of $\bar{C}^{*}$ in Cutler (1993, p. 121) with $b=1$ where $b$ is the number of blocks in Cutler's designs. Hence, in principle, the optimum design problem we consider is the same as that considered in Cutler (1993). However, as mentioned earlier, Cutler (1993) only considered binary block designs whereas we consider nonbinary designs with only one block. In particular, the design used as an illustration in this example is not in the class of designs considered by Cutler (1993).

\subsection{The structure of the information matrix for a general measurement design}

We first introduce some notation and then describe the structure of the information matrix $\boldsymbol{C}$ for a general measurement design. 


\section{Notation.}

$e_{i}=$ the number of times unknown $i$ is the first observation plus the number of times unknown $i$ is the last observation, i.e., $e_{i}=\delta_{1, i}+\delta_{N, i}$. Note that $\sum_{i}^{m} e_{i}=0,1$, or 2 .

$q_{i}=$ the number of times two consecutive observations are of the unknown $i$, i.e., $q_{i}=\sum_{k=1}^{N-1} \delta_{k, i} \delta_{k+1, i}$.

$r_{i, j}=$ the number of times unknowns $i$ and $j$ occur as neighboring pairs in the design.

The following example is given to illustrate these definitions.

Example 2.3. Consider the same sequence of observations as in Example 2.1,

$$
U_{1} U_{3} S U_{1} U_{3} U_{3} U_{3} U_{2} U_{1} U_{2} U_{2} S S,
$$

$e_{1}=1$ (the first observation is of the unknown specimen $U_{1}$ ),

$e_{2}=0$ (neither the first nor the last observation is of $U_{2}$ ),

$e_{3}=0$ (neither the first nor the last observation is of $U_{3}$ ),

$q_{1}=0$ (no two consecutive observations are of $U_{1}$ ),

$q_{2}=1$ (due to observations (10 and 11)),

$q_{3}=2$ (due to the pairs of observations (5 and 6) and (6 and 7)),

$r_{1,2}=2$ (due to the pairs of observations (8 and 9) and (9 and 10)),

$r_{1,3}=2$ (due to the pairs of observations (1 and 2) and (4 and 5)),

$r_{2,3}=1$ (due to observations (7 and 8$)$ ).

The parameters $e_{i}, q_{i}, r_{i j}$ describe the arrangement of observations on the unknowns and the standard and there are certain interrelationships among them which need to be recognized. These interrelationships are summarized in the following Lemma.

Lemma 2.1. Let

$$
t=\frac{1}{m} \sum_{i}^{m} t_{i}, \quad e=\frac{1}{m} \sum_{i}^{m} e_{i}, \quad q=\frac{1}{m} \sum_{i}^{m} q_{i}, \quad r=\frac{1}{\left(\begin{array}{c}
m \\
2
\end{array}\right)} \sum_{i<j}^{m} r_{i, j} .
$$

For any measurement design we must have $N=m t+b-1$. Also, the following relationships among the parameters must hold:

$$
\begin{aligned}
& \left(\begin{array}{c}
m \\
2
\end{array}\right) r+m q=m t-\left(b-b_{0}\right), \\
& \operatorname{maximum}\{2-m e, b-m t\} \leqslant b_{0} \leqslant b-1, \\
& \operatorname{maximum}\left\{0, \frac{m-\left(b-b_{0}\right)}{\left(\begin{array}{c}
m \\
2
\end{array}\right)}\right\} \leqslant r \leqslant \frac{m t-\left(b-b_{0}\right)}{\left(\begin{array}{c}
m \\
2
\end{array}\right)} .
\end{aligned}
$$

The proof is based on simple combinatorial arguments and is omitted.

The following lemma describes the elements of the information matrix $\boldsymbol{C}$ corresponding to an arbitrary measurement design. 
Lemma 2.2. Let $\mathscr{D}$ denote an arbitrary measurement design described by the parameters $e_{i}, q_{i}, t_{i}, b_{0}, b, m$ and $N$. Let $\boldsymbol{C}$ denote the information matrix for $\tau$ under the design $\mathscr{D}$. Then the elements of $\boldsymbol{C}=\left(C_{i j}\right), i, j=1,2, \ldots, m$ are given by

$$
C_{i, j}= \begin{cases}g_{i}-(1-\phi) s_{i}^{2} / h & \text { if } i=j, \\ -r_{i, j} \phi-(1-\phi) s_{i} s_{j} / h & \text { if } i \neq j,\end{cases}
$$

where

$$
\begin{aligned}
& g_{i}=t_{i}\left(1+\phi^{2}\right)-2 q_{i} \phi-e_{i} \phi^{2}, \\
& s_{i}=t_{i}(1-\phi)+e_{i} \phi, \\
& h=2+\left(\sum_{i}^{m} t_{i}+b-3\right)(1-\phi) .
\end{aligned}
$$

It is interesting to note that the information matrix in Lemma 2.2 has the same structure as the one given in Kunert and Martin (1987, p. 1606) except that the value of $N$ in our problem is $m t+b-1$ and in their problem it is $m t$. However, the information matrix $C$ of Lemma 2.2 is in general nonsingular whereas the matrix $\mathscr{C}_{d}$ of Kunert and Martin (1987) is always singular. There appears to be no useful connection between our problem and theirs which allows us to obtain optimum designs for our problem.

The following lemma describes conditions that must be satisfied by the design parameters $e_{i}, q_{i}, t_{i}$, and $r_{i j}$ in order for a measurement design to be balanced. It also gives the information matrix corresponding to balanced designs.

Lemma 2.3. A measurement design is balanced for all values of $\phi(-1<\phi<1)$ if and only if, for all $i$ and $j, t_{i}=t, q_{i}=q, e_{i}=e$, and $r_{i, j}=r$. When this holds, the matrix $\boldsymbol{C}$ is completely symmetric and is equal to $x \boldsymbol{I}_{m}+y \boldsymbol{J}_{m}$, where

$$
\begin{aligned}
& x=t(1-\phi)^{2}+\frac{2\left(b-b_{0}\right)}{m} \phi+m r \phi-e \phi^{2}, \\
& y=-r \phi-\frac{(1-\phi)(t(1-\phi)+e \phi)^{2}}{2+(N-2)(1-\phi)},
\end{aligned}
$$

and the trace of $\boldsymbol{C}^{-1}$ can be explicitly expressed as

$$
\operatorname{Trace}\left(\boldsymbol{C}^{-1}\right)=\frac{m-1}{x}+\frac{1}{x+m y} \text {. }
$$

The proof involves straightforward algebra and is omitted.

According to the above lemma, $e_{i}$ must all be equal to a common value $e$ for a balanced design. Also, the sum of the $e_{i}$ 's cannot be greater than 2 . Therefore, when $m \geqslant 3$, each $e_{i}$ must be zero, i.e., $e$ must equal zero. Observe that the eigenvalues of the matrix $C$ are $x+m y$ with multiplicity one and $y$ with multiplicity $m-1$. These may be obtained by substituting $b=1$ in Cutler (1993) formulas for the eigenvalues of $\bar{C}^{*}$ under a rectangular $A R(1)$ error structure.

The following lemma gives a necessary and sufficient condition for the existence of a balanced measurement design corresponding to specified values of $m, b$, and $t$. 
Lemma 2.4. Suppose $m \geqslant 3$. A balanced measurement design exists corresponding to specified values of $m, b$, and $t$, if and only if there exist nonnegative integers $q$ and $r$ with $0 \leqslant q \leqslant t-1$ such that $2+m t-b \leqslant m q+m(m-1) r / 2 \leqslant m t-1$.

The necessity follows from (2.7)-(2.9) using the fact that $e=0$ when $m \geqslant 3$, and the requirement for $q, r, b_{0}$ to be nonnegative integers satisfying the conditions $0 \leqslant q \leqslant t-1$ and $2 \leqslant b_{0} \leqslant b-1$. The sufficiency follows by noting that the algorithm for constructing balanced designs, given in Section 4, is applicable whenever the condition of Lemma 2.4 is met.

For given values of $N$ and $m$ it is often possible to enumerate, by the use of Lemma 2.4, the entire collection of balanced designs. This is illustrated in Examples 2.4 and 2.5 .

Example 2.4. Suppose we wish to identify the collection of balanced designs when there are $m=20$ unknown specimens to be measured and the total number of observations that can be made using available resources is $N=60$. By Lemma 2.4, we need to determine the collection of integer solutions to the inequalities

$$
20 t-b+2 \leqslant 20 q+190 r \leqslant 20 t-1
$$

satisfying $20 t+b-1=60$ and nonnegativity restrictions. The value of $t$ must be at least 1 . If we take $t=1$ then $b$ must equal 41 and the inequality in (2.14) becomes

$$
-19 \leqslant 20 q+190 r \leqslant 19
$$

which has only one solution, namely $q=0$ and $r=0$. If we take $t=2$ then $b=21$ and the condition in (2.14) becomes

$$
21 \leqslant 20 q+190 r \leqslant 39
$$

which has no feasible solutions. No other solutions are feasible, so there is only one balanced design corresponding to $N=60$ and $m=20$. It has parameters $b=41$, $t=1, q=0, r=0, e=0$, and $b_{0}=21$.

Example 2.5. Now consider the case $N=60$ and $m=4$. Using the conditions in Lemma 2.4 it is easily verified that there are 204 balanced designs in all. In particular, there are 26 designs corresponding to the value $t=10$. We will return to this example later in Section 3.

In some problems it will be relatively straightforward to determine the optimum balanced design, given the value of the $\mathrm{AR}(1)$ parameter $\phi$, simply by enumerating the entire collection of balanced designs corresponding to the given values of the design parameters, computing the trace criterion for each design in the collection, and determining the best among them. There is a serious disadvantage to this approach. It requires that the value of $\phi$ be specified in advance. If one is interested in examining optimum balanced designs for a range of values of $\phi$ then the numerical optimization must be carried out over a grid of $\phi$ values with respect to the enumerated collection 
of balanced designs. Alternatively, Theorems 3.1 and 3.2 given in the next section may be used to prune the list of balanced designs to a shorter list of candidate designs for optimality. Details of this approach will be given later in this article.

Kiefer (1975) provided a methodology which can be used to find optimal designs for various classes of problems. A key step in his approach depends on the convexity of the trace of the inverse of a positive-definite matrix. The following result, which is a consequence of a well known convexity property of positive-definite matrices (see Theorem 1.1.12 of Fedorov (1972)), supplies the condition needed to apply Kiefer's method.

Lemma 2.5. For a given information matrix $\boldsymbol{C}$ described in (2.10), we have $\operatorname{Trace}(\overline{\boldsymbol{C}})^{-1} \leqslant \operatorname{Trace}\left(\boldsymbol{C}^{-1}\right)$

where $\overline{\boldsymbol{C}}=\left(\bar{C}_{i, j}\right)$ with

$$
\bar{C}_{i, j}= \begin{cases}\frac{2}{m(m-1)} \sum_{k<l} C_{k, l} & \text { for } i \neq j \\ \frac{1}{m} \sum_{k=1}^{m} C_{k, k} & \text { for } i=j .\end{cases}
$$

Clearly, $\overline{\boldsymbol{C}}$ is of the form $x \boldsymbol{I}_{m}+y \boldsymbol{J}_{m}$ where $\boldsymbol{I}_{m}$ is an identity matrix, $\boldsymbol{J}_{m}$ is a $m \times m$ matrix with all elements equal to 1 . By virtue of Lemma 2.5 it follows that a design that is optimum within the class of balanced designs is sometimes optimum within the class of all feasible designs. This is the essence of the following corollary.

Corollary 2.6. Suppose $-1<\phi<1$ and positive integers $N$ and $m(N>m)$ are given quantities. Let $t, e, q$, and $r$ be nonnegative real numbers satisfying the conditions given in (2.7)-(2.9). Let $\sigma_{t}$ and $\sigma_{e}$ be nonnegative real numbers and $-1 \leqslant \rho \leqslant 1$. Define $y^{*}$ and $x^{*}$ by

$$
\begin{aligned}
y^{*}= & -\phi r-\frac{m(1-\phi)}{(m-1)[N-(N-2) \phi)]}\{t(1-\phi)+e \phi\}^{2} \\
& +\frac{(1-\phi)^{3}}{(m-1)[N-(N-2) \phi]}\left\{t^{2}+\sigma_{t}^{2}\right\} \\
& +\frac{\phi^{2}(1-\phi)}{(m-1)[N-(N-2) \phi]}\left\{e^{2}+\sigma_{e}^{2}\right\}+\frac{2 \phi(1-\phi)^{2}}{(m-1)[N-(N-2) \phi]}\left\{e t+\rho \sigma_{t} \sigma_{e}\right\}
\end{aligned}
$$

and

$$
\begin{aligned}
x^{*}= & t\left(1+\phi^{2}\right)-2 q \phi-e \phi^{2}-\frac{(1-\phi)}{[N-(N-2) \phi)]}\left\{(1-\phi)^{2}\left(t^{2}+\sigma^{2}\right)+\phi^{2}\left(e^{2}+\sigma_{e}^{2}\right)\right. \\
& \left.+2 \phi(1-\phi)\left(e t+\rho \sigma_{t} \sigma_{e}\right)\right\}-y^{*}
\end{aligned}
$$

Over the space of allowable values of $t, e, q, r, \sigma_{t}, \sigma_{e}$, and $\rho$ for which the matrix $x^{*} \boldsymbol{I}_{m}+y^{*} \boldsymbol{J}_{m}$ is positive definite, suppose the minimum value of the trace of $\left(x^{*} \boldsymbol{I}_{m}+\right.$ 
$\left.y^{*} \boldsymbol{J}_{m}\right)^{-1}$ occurs at $t=t_{0}, e=e_{0}, r=r_{0}, q=q_{0}, \sigma_{t}=0$, and $\sigma_{e}=0$, where $t_{0}, e_{0}, q_{0}, r_{0}$ are integers. Then, a balanced measurement design with parameters $N, m, t_{0}, q_{0}, r_{0}, e_{0}$ is A-optimum over the class of all measurement designs with $m$ unknowns and $N$ observations.

The proof follows by expressing the entries of the matrix $\bar{C}$ in terms of the parameters for a general measurement design with $m$ unknowns and $N$ observations. Details are omitted. Corollary 2.6 may also be used to obtain a lower bound for the trace criterion for an arbitrary measurement design by carrying out the minimization of the trace without requiring the design parameters to be integers. This lower bound can then be used to judge the efficiency of an optimum balanced design. This is illustrated in Section 3 .

\section{A-optimum balanced designs for three or more unknowns}

The determination of an A-optimum design in the class of all balanced designs is a discrete optimization problem with inequality constraints. Different optimum designs are obtained for different ranges of values of $\phi$. In this section we derive the optimum balanced designs when the values of $N, m, t$, and $\phi$ are specified. The results can be used to develop a computer algorithm for finding the best value of $t$ and the corresponding optimum design for specified $N, m$, and $\phi$. The computer implementation is aided by observing that, for fixed $N, m$, and $\phi$, the function

$$
T(t)=\underset{q, r, b_{0}}{\operatorname{Min}} \operatorname{Trace}\left(\boldsymbol{C}^{-1}\right)
$$

is a convex function of $t$.

We first observe that an explicit expression for $\operatorname{Trace}\left(\boldsymbol{C}^{-1}\right)$ for balanced designs with $m \geqslant 3$ is given by

$$
\begin{aligned}
\operatorname{Trace}\left(\boldsymbol{C}^{-1}\right)= & \frac{m-1}{t(1-\phi)^{2}+\frac{2\left(b-b_{0}\right) \phi}{m}+m r \phi} \\
& +\frac{1}{t(1-\phi)^{2}+\frac{2\left(b-b_{0}\right) \phi}{m}-\frac{m t^{2}(1-\phi)^{3}}{2+(N-2)(1-\phi)}} .
\end{aligned}
$$

For fixed values of $m$, and $N$, the right-hand side of (3.1) may be regarded as a function of $b_{0}, t$, and $r$. We minimize this function with respect to $b_{0}$ and $r$ for each allowable value of $t$, thus finding optimal balanced designs for each allowable combination of values of $b$ and $t$. The global optimum balanced design is then the best design among the set of optimum balanced designs obtained for the different admissible values of $b$ and $t$. We state the following two results, one for the case $-1<\phi<0$ and the other for the case $0<\phi<1$. Proofs are given in the appendix.

Theorem 3.1. Suppose $m$, the number of unknown specimens, is greater than or equal to 3 and $-1<\phi<0$, where $\phi$ is the parameter in the AR(1) model for the errors. 
Suppose each unknown specimen is to be measured times and that the total number of measurements, including those on the standard, is $N$. The values of $b_{0}$ and $r$ corresponding to an optimal balanced design are as follows:

(1) If $b-2 \geqslant m$ and $m$ is odd, then $b_{0}=b-m, r=0$ and $q=t-1$.

(2) If $b-2 \geqslant m$ and $m$ is an even number greater than $2 t$, then $b_{0}=b-m, r=0$, and $q=t-1$.

(3) If $b-2 \geqslant m$ and $m$ is an even number less than or equal to $2 t$, then the balanced optimum design is obtained from one of the following two possibilities:

(3a) $b_{0}=b-m, r=0$, and $q=t-1$, or (3b) $b_{0}=b-m / 2, r=1$, and $q=t-\frac{1}{2} m$.

The choice is to be made by numerically comparing the average variances corresponding to each of these cases and the result can depend on the value of $\phi$.

(4) If $m / 2 \leqslant b-2<m$ and $m$ is an even integer less than or equal to $2 t$, then $b_{0}=b-m / 2, r=1$, and $q=t-\frac{1}{2} m$.

(5) In all other cases balanced designs do not exist.

Theorem 3.2. Suppose $m$, the number of unknown specimens, is greater than or equal to 3 and $0<\phi<1$ where $\phi$ is the AR(1) parameter in the model for the errors. Suppose each unknown specimen is to be measured times and that the total number of measurements, including those on the standard, is $N$. The values of $b_{0}, q$ and $r$ corresponding to an optimal balanced design are as follows:

(1) If $m$ is even and $\frac{1}{2} \leqslant(b-2) / m \leqslant t$, then the optimum balanced design is obtained from one of the following four possibilities. The four possible designs given below must be numerically compared to pick the best among them. The result will generally depend on the value of $\phi$.

(1a) Suppose the interval given by

$$
\text { maximum }\left\{0, \frac{2(m-b+2)}{m(m-1)}\right\} \leqslant r \leqslant \frac{2(m t-b+2)}{m(m-1)}
$$

contains at least one even nonnegative integer. Then a candidate for an optimum balanced design has $b_{0}=b-m x_{0}$ and $r=r_{0}$ where $r_{0}$ is the largest even integer in the interval in (3.2) and $x_{0}$ is the largest positive integer less than or equal to $(b-2) / m$. Note that this case can occur only when $(b-2) / m \geqslant 1$.

(1b) Suppose the interval in (3.2) contains at least one nonnegative odd integer. Then a candidate for an optimum balanced design has $b_{0}=b-m x_{0}$ and $r=r_{0}$ where $r_{0}$ is the largest odd integer in the interval given by (3.2), and $x_{0}$ is the largest positive odd multiple of $1 / 2$ less than or equal to $(b-2) / \mathrm{m}$.

(1c) Suppose the interval given by

$$
\frac{2(m t-b+2)}{m(m-1)}<r \leqslant \frac{2(m t-1)}{m(m-1)}
$$

contains at least one nonnegative even integer. Then a candidate for an optimum balanced design has $b_{0}=b-m x_{0}$ and $r=r_{0}$ where $r_{0}$ is the smallest even integer in the interval given by (3.3) and

$$
x_{0}=t-(m-1) r_{0} / 2 \text {. }
$$


(1d) Suppose the interval in (3.3) contains at least one odd integer. Then a candidate optimum balanced design has $b_{0}=b-m x_{0}$ and $r=r_{0}$ where $r_{0}$ is the smallest odd integer in the interval in (3.3) and

$$
x_{0}=t-(m-1) r_{0} / 2 .
$$

(2) If $m$ is odd and $1 \leqslant(b-2) / m \leqslant t$. Then the optimum design is given by one of the following two possibilities. They must be numerically compared and the better one must be chosen. The result will generally depend on the value of $\phi$.

(2a) Suppose the interval in (3.2) contains at least one nonnegative integer. Then a candidate optimum balanced design has $b_{0}=b-m x_{0}$ and $r=r_{0}$ where $r_{0}$ is the largest integer in the interval given by (3.2), and $x_{0}$ is the largest integer less than or equal to $(b-2) / m$.

(2b) Suppose the interval in (3.3) contains at least one nonnegative integer. Then a candidate optimum balanced design has $b_{0}=b-m x_{0}$ and $r=r_{0}$ where $r_{0}$ is the smallest integer in the interval given by (3.3) and $x_{0}=t-(m-1) r_{0} / 2$.

(3) $(b-2) / m>t$, then the optimum balanced design has $b_{0}=b-m t$ and $r=0$.

(4) In all remaining cases a balanced design does not exist.

Example 3.1. We will use Theorems 3.1 and 3.2 to make a complete determination of optimum balanced designs over the entire range $-1<\phi<1$ of values of $\phi$, for the case when $N=60, m=4, t=10$, and $b=21$. This is a subclass of designs considered in Example 2.4.

First consider the case $\phi<0$. Observing that $b-2=19$ is greater than $m=4$ and that $m$ is an even number less than $2 t=20$, we decide we are in case (3) of Theorem 3.1. Therefore there are two candidates for an optimum balanced design, say $D_{1}$ and $D_{2}$, given by cases (3a) and (3b), respectively. For design $D_{1}$ we must take $b_{0}=b-m=17$ and $r=0$. For design 2 we must choose $b_{0}=b-m / 2=19$ and $r=1$. The complete set of design parameters for the two designs is listed below.

$$
\text { Design } D_{1}: \quad N=60, m=4, b=21, t=10, q=9, r=0, b_{0}=17 \text {, }
$$

Design $D_{2}: \quad N=60, m=4, b=21, t=10, q=8, r=1, b_{0}=19$.

Let $\operatorname{tr}_{1}$ and $\operatorname{tr}_{2}$ denote the trace criterion for $D_{1}$ and $D_{2}$, respectively. We have

$$
\operatorname{tr}_{1}=\frac{2\left(-75+190 \phi-186 \phi^{2}+70 \phi^{3}\right)}{\left(5-9 \phi+5 \phi^{2}\right)\left(-50+115 \phi-111 \phi^{2}+45 \phi^{3}\right)}
$$

and

$$
\operatorname{tr}_{2}=\frac{8\left(-75+190 \phi-186 \phi^{2}+70 \phi^{3}\right)}{5\left(2-3 \phi+2 \phi^{2}\right)\left(-100+260 \phi-251 \phi^{2}+90 \phi^{3}\right)} .
$$

These are rational functions of $\phi$ and it is easy to show that $\operatorname{tr}_{2} \leqslant \operatorname{tr}_{1}$ for all $-1<\phi<0$. So design $D_{2}$ is optimum for all negative values of $\phi$. Fig. 1 shows the graphs of $\operatorname{tr}_{1}$ and $\operatorname{tr}_{2}$ as functions of $\phi$ in the interval $-1 \leqslant \phi \leqslant 0$. The two curves appear indistinguishable in the figure. 


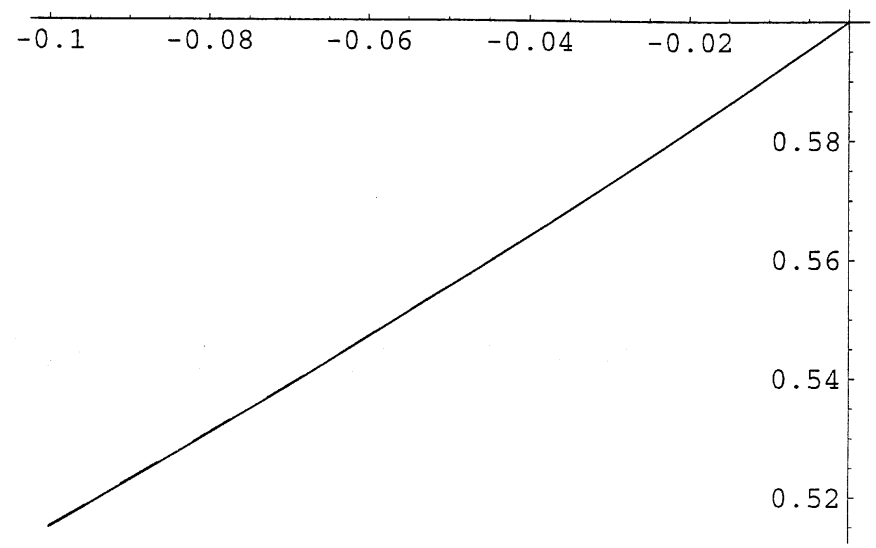

Fig. 1. Plot of the trace criterion for designs $D_{1}$ (dotted curve) and $D_{2}$ (solid curve).

Next, consider the case $\phi>0$. Since $m=4$ is even and $(b-2) / m=4.75$ is between $1 / 2$ and $t=10$, case (1) holds. We examine the four subcases for this situation. For (1a) we note that the interval for $r$ in (3.2) is $0 \leqslant r \leqslant 21 / 6$, so $r_{0}=2$ is the largest even integer in this interval. Also $x_{0}=4$. Hence, $b_{0}=b-m x_{0}=5$ and $q=3$. So case (1a) leads to the design $D_{3}$ with the parameters listed below.

Design $D_{3}: \quad N=60, m=4, b=21, t=10, q=3, r=2, b_{0}=5$.

Under case (1b) we have $r_{0}=3$ and $x_{0}=9 / 2$. So case (1b) leads to the design $D_{4}$ with the parameters listed below.

Design $D_{4}: \quad N=60, m=4, b=21, t=10, q=1, r=3, b_{0}=3$.

The interval for $r$ in (3.3) is $21 / 6<r \leqslant 39 / 6$, so we get the following candidate designs under case (1c) and case (1d):

Design $D_{5}: \quad N=60, m=4, b=21, t=10, q=0, r=4, b_{0}=5$,

Design $D_{6}: \quad N=60, m=4, b=21, t=10, q=0, r=5, b_{0}=11$.

Denote the trace criterion for designs $D_{3}, D_{4}, D_{5}, D_{6}$ by $\operatorname{tr}_{3}, \operatorname{tr}_{4}, \operatorname{tr}_{5}, \operatorname{tr}_{6}$, respectively. We have

$$
\begin{aligned}
& \operatorname{tr}_{3}=\frac{10\left(-15+14 \phi-14 \phi^{2}+14 \phi^{3}\right)}{\left(5-2 \phi+5 \phi^{2}\right)\left(-50+25 \phi-24 \phi^{2}+45 \phi^{3}\right)}, \\
& \operatorname{tr}_{4}=\frac{8\left(-75+40 \phi-41 \phi^{2}+70 \phi^{3}\right)}{\left(10+\phi+10 \phi^{2}\right)\left(-100+20 \phi-19 \phi^{2}+90 \phi^{3}\right)}, \\
& \operatorname{tr}_{5}=\frac{2\left(-75+40 \phi-41 \phi^{2}+70 \phi^{3}\right)}{\left(5+2 \phi+5 \phi^{2}\right)\left(-50+25 \phi-24 \phi^{2}+45 \phi^{3}\right)}
\end{aligned}
$$




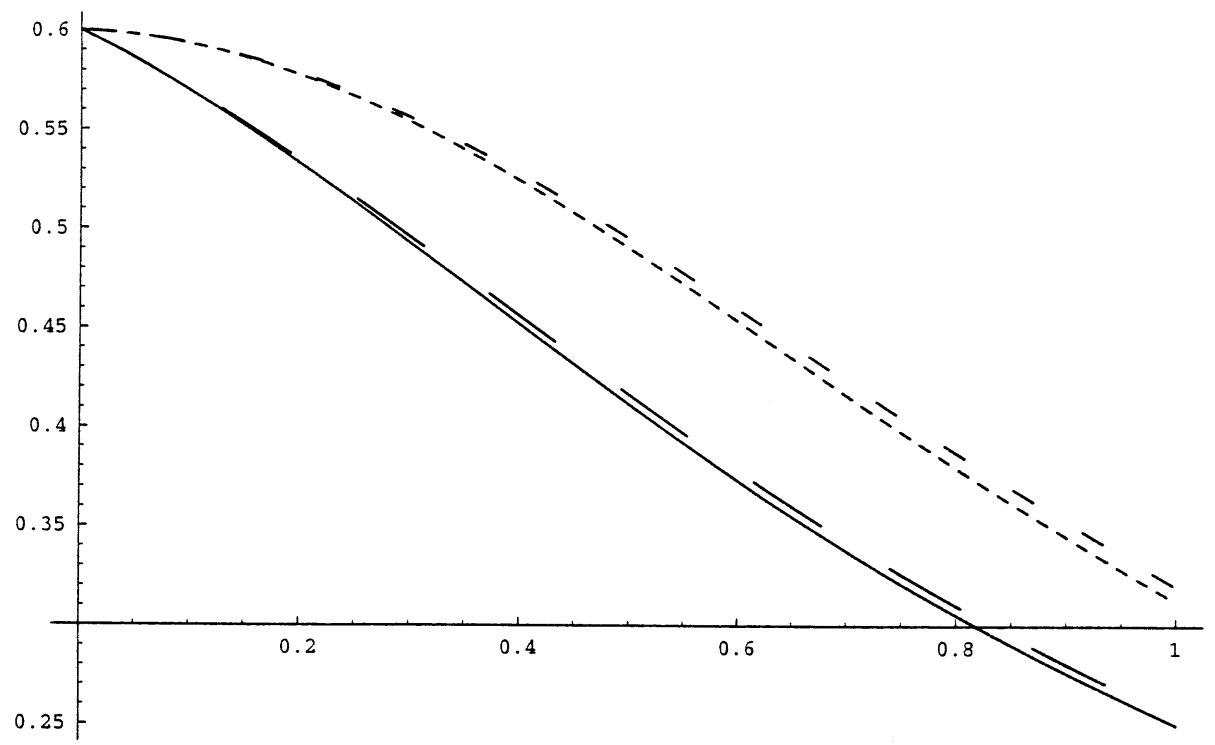

Fig. 2. Plot of the trace criterion for designs $D_{3}$ (dotted curve), $D_{4}$ (dashed curve), $D_{5}$ (solid curve) and $D_{6}$ (dot-dash curve).

and

$$
\operatorname{tr}_{6}=\frac{8\left(-15+14 \phi-14 \phi^{2}+14 \phi^{3}\right)}{5\left(2+\phi+2 \phi^{2}\right)\left(-20+28 \phi-27 \phi^{2}+18 \phi^{3}\right)} .
$$

Again, since these are rational functions of $\phi$, it is easy to show that $\operatorname{tr}_{5}$ is smaller than $\operatorname{tr}_{3}, \operatorname{tr}_{4}$ and $\operatorname{tr}_{6}$ over the interval $0<\phi<1$. Therefore $D_{5}$ is the optimum design for any positive value of $\phi$. Fig. 2 shows the graphs of $\operatorname{tr}_{3}, \operatorname{tr}_{4}, \operatorname{tr}_{5}$ and $\operatorname{tr}_{6}$ as functions of $\phi$ in the interval $0 \leqslant \phi \leqslant 1$.

The use of Theorems 3.1 and 3.2 has enabled us to determine that design $D_{2}$ is optimum in the class of balanced designs with $m=4, t=10, b=21$ (there are 26 of these designs) for every value of $\phi$ between -1 and 0 . Likewise, we have determined that design $D_{5}$ is optimum in this class for every value of $\phi$ between 0 and 1 (when $\phi=0$, every balanced design with these parameters leads to the same trace criterion).

It may be verified that, for $N=60, m=4$, and $\phi=0.1$, the trace of $\left(x^{*} \boldsymbol{I}+y^{*} \boldsymbol{J}\right)^{-1}$ in Corollary 2.5 has a minimum value equal to 0.559 and the corresponding value for $t$ is 9.86 (rounded to two decimals). So a lower bound for the trace criterion for an arbitrary measurement design is equal to 0.559 when $N=60, m=4$, and $\phi=0.1$. The optimum balanced design $D_{5}$ above has a trace criterion equal to 0.5698 at $\phi=0.1$ which is very close to the lower bound. 


\section{An algorithm to construct balanced measurement designs}

For given values of $m, N$, and $t$, we gave in the previous section the values of $r$ and $b_{0}$ which minimize $\operatorname{Trace}\left(\boldsymbol{C}^{-1}\right)$. The parameters values obtained from Theorems 3.1 and 3.2 automatically satisfy the necessary conditions for balance, stated in Lemma 2.5. It remains to show how to construct the desired balanced designs corresponding to $m$, $t, r$ and $b_{0}$.

For our construction, we will need to use a special class of latin squares which were considered by Kiefer and Wynn (1981) in their study of equineighbored designs. Also see Cheng (1983). For a given integer $m$, consider the $m \times m$ array $\boldsymbol{A}$ whose $(j, l)$ element $(1 \leqslant j, l \leqslant m)$ is the symbol $U_{p}$ where

$$
p-1=\left[\sum_{i=1}^{j}(-1)^{i}(i-1)+\sum_{i=1}^{l}(-1)^{i}(i-1)\right]_{\bmod m} .
$$

It is easily verified that $\boldsymbol{A}$ has the following properties:

(1) $\boldsymbol{A}$ is a latin square. Each column or each row of $\boldsymbol{A}$ contains all $m$ different symbols (which represent the $m$ unknown specimens) exactly once.

(2) When $m$ is an odd number, $\boldsymbol{A}$ is symmetric. When $m$ is an even number, then $\boldsymbol{A}$ is symmetric with respect to the two leading diagonals, i.e., $a_{j, l}=a_{l, j}=a_{m+1-j, m+1-l}=$ $a_{m+1-l, m+1-j}$.

(3) For $m$ odd, each pair of symbols appear as neighbors exactly once in the rows of the first $m \times(m+1) / 2$ subarray.

(4) For $m$ even, each pair of symbols appear as neighbors exactly once in the rows of the first $(m / 2) \times m$ subarray and exactly twice in the rows of the entire array.

The following arrays are given to illustrate these results.

Example 4.1. For $m=5$ and 6, the arrays obtained from (4.1) are, respectively,

$$
\begin{aligned}
& \begin{array}{llllll}
U_{5} & U_{1} & U_{4} & U_{2} & U_{3}
\end{array} \\
& \begin{array}{lllll}
U_{1} & U_{2} & U_{5} & U_{3} & U_{4}
\end{array} \\
& \begin{array}{llllll}
U_{4} & U_{5} & U_{3} & U_{1} & U_{2}
\end{array} \\
& \begin{array}{lllll}
U_{2} & U_{3} & U_{1} & U_{4} & U_{5}
\end{array} \\
& \begin{array}{llllll}
U_{3} & U_{4} & U_{2} & U_{5} & U_{1}
\end{array} \\
& \begin{array}{llllll}
U_{6} & U_{1} & U_{5} & U_{2} & U_{4} & U_{3} \\
U_{1} & U_{2} & U_{6} & U_{3} & U_{5} & U_{4} \\
U_{5} & U_{6} & U_{4} & U_{1} & U_{3} & U_{2} \\
U_{2} & U_{3} & U_{1} & U_{4} & U_{6} & U_{5} \\
U_{4} & U_{5} & U_{3} & U_{6} & U_{2} & U_{1} \\
U_{3} & U_{4} & U_{2} & U_{5} & U_{1} & U_{6}
\end{array}
\end{aligned}
$$

Based on the arrays produced by (4.1), we present the following algorithm to construct balanced measurement designs. We consider three separate cases: (A) $r=0$, (B) $r>0$ with $(m-1) r$ odd, and (C) $r>0$ with $(m-1) r$ is even.

\section{The Algorithm}

Case (A): Suppose $r=0$. Then $m q=m t-\left(b-b_{0}\right)$ and $k=\left(b-b_{0}\right) / m$ must be an integer. In particular $q=t-k$. Let $t=u k+v$ where $u$ and $v$ are integers and $0 \leqslant v<k$. Construct an array $\boldsymbol{D}$ (not necessarily a rectangular array), with $m k$ rows such that, for each $i$ with $1 \leqslant i \leqslant m$, there are $k-v$ rows consisting only of $u$ occurrences of $U_{i}$ and $v$ rows consisting only of $u+1$ occurrences of $U_{i}$. To this array, add an 
initial column consisting of $m k$ standards, and also add a single standard after the last element of the last row. Concatenate all the rows of this array to produce a single row consisting of $m(k-v) u+m v(u+1)+m k+1=N$ symbols. If $b_{0}=2$, then this is the required measurement design. If $b_{0}>2$ then add $b_{0}-2$ additional standards to the current design sequence by placing each additional standard next to an already occurring standard. This can usually be done in many different ways and each one will lead to a design satisfying the requirements.

Case (B): Suppose $r>0$ and $(m-1) r$ is an even integer. Thus either $m$ is odd or $m$ and $r$ are both even. We must have $b-b_{0}=k m$ for some integer $k$. The following steps lead to a balanced measurement design meeting the requirements and with (nearly) equal batch sizes for the nonempty batches.

Step (1): For a given $m$, let $\boldsymbol{A}$ be the $m \times m$ array obtained from (4.1). If $m$ is odd, then let $m_{1}=(m+1) / 2$, otherwise $m_{1}=m / 2$. Let $\boldsymbol{A}_{1}$ be the first $m \times m_{1}$ subarray of $\boldsymbol{A}$ and $\boldsymbol{A}_{2}$ be the $m \times m_{1}$ array which is the "reflection" of $\boldsymbol{A}_{1}$, i.e., $\boldsymbol{A}_{2}$ is obtained by permuting the columns of $\boldsymbol{A}_{1}$ according to the permutation

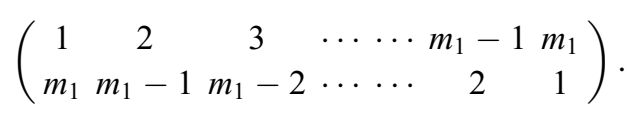

Now we define an operation for merging two arrays by columns. Let $\boldsymbol{B}=\boldsymbol{B}_{1} \vdots \boldsymbol{b}$ and $\boldsymbol{C}=\boldsymbol{c}: \boldsymbol{C}_{1}$ be two arrays of sizes $m_{0} \times l_{1}$ and $m_{0} \times l_{2}$, respectively, and $\boldsymbol{b}$ and $\boldsymbol{c}$ are column vectors. Then the operation $\circ$ is defined by

$$
\boldsymbol{B} \circ \boldsymbol{C}= \begin{cases}\boldsymbol{B}_{1} \vdots \boldsymbol{b} \vdots \boldsymbol{C}_{1} & \text { if } \boldsymbol{b}=\boldsymbol{c}, \\ \boldsymbol{B}_{1} \vdots \boldsymbol{b} \vdots \boldsymbol{c} \vdots \boldsymbol{C}_{1} & \text { if } \boldsymbol{b} \neq \boldsymbol{c} .\end{cases}
$$

Let the array $\boldsymbol{D}_{1}=\boldsymbol{A}_{1}$. If $r>1$, then for $j \geqslant 2$ recursively define $\boldsymbol{D}_{j}=\boldsymbol{D}_{j-1} \circ \boldsymbol{A}_{1}$ if $j$ is odd and $\boldsymbol{D}_{j}=\boldsymbol{D}_{j-1} \circ \boldsymbol{A}_{2}$ if $j$ is even. By using Properties (3) and (4) of $\boldsymbol{A}$ described previously and mathematical induction, it is easy to verify that each pair of unknown specimens appears as neighbors exactly $r$ times in the $m$ rows of the array $\boldsymbol{D}_{r}$. Furthermore, no unknown occurs next to itself in any of the $m$ rows. Note also that $\boldsymbol{D}_{r}$ is an array of size $m \times n_{1}$, where $n_{1}=1+(m-1) r / 2$.

Step (2): If $q$ is equal to 0 then go to Step (3). Suppose $q$ is greater than 0 . Let $\boldsymbol{c}_{1}, \ldots, \boldsymbol{c}_{n_{1}}$ be the $n_{1}$ columns of $\boldsymbol{D}_{r}$. Enlarge the current array $D_{r}$ by repeating selected columns of $D_{r}$ next to themselves such that the number of columns in the enlarged array is $q+n_{1}=q+1+(m-1) r / 2$. It makes no difference as to which columns are selected to be repeated or how many times a given column is repeated as long as the total number of new columns added is $q$. At the end of this step each unknown specimen occurs in the array exactly $n=q+1+(m-1) r / 2$ times. This follows from the fact that each column of $D_{r}$ contains each unknown exactly once.

Step (3): If $k=1$ then go to Step (4). Suppose $k>1$. Let $k_{0}=k-1$. Consider integers

$$
1 \leqslant i_{1}<i_{2}<\cdots<i_{k_{0}-1}<i_{k_{0}} \leqslant n .
$$


For each $j=1, \ldots, k_{0}$, replace column $\boldsymbol{c}_{i_{j}}$ in $\boldsymbol{D}_{r}$ by the block of three columns $\boldsymbol{l}$ arge $\boldsymbol{c}_{i_{j}}|\boldsymbol{s}| \boldsymbol{c}_{i_{j}}$ where $\boldsymbol{s}$ is a column of $S$ 's representing measurements of a standard. The resulting modified array $\boldsymbol{D}_{r}$ will now have $n+k-1=t$ columns consisting of unknown specimens and $k-1$ columns consisting of standards only.

Step (4): Add a column $\boldsymbol{s}$ of standards as the first row of $\boldsymbol{D}_{r}$ and also add a single standard to the right of the last element of the last row. The total number of standards in this modified (nonrectangular) array is $m k+1=(b-1)-\left(b_{0}-2\right)$. Concatenate the rows of this array to produce a single row consisting of standards and unknowns. If $b_{0}=2$, then this row, when viewed as a measurement design, meets all the requirements. If $b_{0}>2$ then the number of standards occurring in the current array $\boldsymbol{D}_{r}$ is less than the required number by an amount equal to $b_{0}-2$ as is the number of empty batches. This can be rectified by including $b_{0}-2$ additional standards such that each of the added standard is placed adjacent to an already occurring standard. This also creates $b_{0}-2$ additional empty batches. This final row consisting of unknowns and standards is then a balanced measurement design meeting all the requirements.

Note: The batch sizes in this design depend on how the columns $i_{1}<\cdots<i_{k_{0}}$ are chosen. Let $t=(k-1) u+v$ where $u$ and $v$ are nonnegative integers and $|u-v|$ is as small as possible. For $j=1, \ldots, k_{0}$, choose $i_{j}=j u-j+1$. Then the resulting measurement design will have $(k-1) m$ batches of size $u$ and $m$ batches of size $v$ and thus will have nearly equal batch sizes. Other choices of $i_{1}, \ldots, i_{k_{0}}$ are also possible.

Case (C): Now suppose $r>0$ and $(m-1) r$ is odd. In this case $b-b_{0}=k m / 2$, where $k$ is an odd positive integer. The following steps will lead to a (nearly) equal batch-size design.

Step (1): Let $\boldsymbol{A}$ be the $m \times m$ array obtained from (4.1). Let $\boldsymbol{A}_{1}$ be the $m / 2 \times m$ array of $\boldsymbol{A}$ consisting of the first $m / 2 \times m$ subarray of $\boldsymbol{A}$. Also let $\boldsymbol{A}_{2}$ be the $m / 2 \times m$ array that is the "reflection" of $A_{1}$ as described earlier.

Let the array $\boldsymbol{D}_{1}=\boldsymbol{A}_{1}$. If $r>1$, then for $j \geqslant 2$ define $\boldsymbol{D}_{j}$ recursively by $\boldsymbol{D}_{j}=\boldsymbol{D}_{j-1} \circ \boldsymbol{A}_{1}$ if $j$ is odd, and $\boldsymbol{D}_{j}=\boldsymbol{D}_{j-1} \circ \boldsymbol{A}_{2}$ if $j$ is even. Each pair of unknown specimens appears as neighbors exactly $r$ times in the $m / 2$ rows of the array $\boldsymbol{D}_{r}$. Also $\boldsymbol{D}_{r}$ is an array of size $m / 2 \times n_{1}$, where $n_{1}=m+(m-1)(r-1)$.

Step (2): If $q$ equals zero, then go to Step (3). Suppose $q>0$. By using Properties (1) and (2) of the array $\boldsymbol{A}$ described previously, it is easily checked that for each $i$, $i=1, \ldots, n_{1} / 2$, the pair of columns, column $i$ and column $n_{1}+1-i$, together contains all $m$ different unknown specimens exactly once. Now enlarge the current array $\boldsymbol{D}_{r}$ by repeating next to themselves selected pairs of columns, column $i$ and column $n_{1}+1-i$ for various $i$, such that the total number of additional pairs of columns added is $q$. It makes no difference which pairs of columns are selected to be repeated or how many times a given pair of columns is repeated so long as the total number of added pairs is $q$.

At this point the array $\boldsymbol{D}_{r}$ has $m / 2$ rows and $n=n_{1}+2 q$ columns. We also note that, for each $i$, the unknown $U_{i}$ occurs next to itself exactly $q$ times among its rows. Moreover, each unknown occurs in the array exactly $q+n_{1} / 2=q+m / 2+(m-1)(r-1) / 2$ 
times. Since $t=q+n_{1} / 2+(k-1) / 2$ we need $(k-1) / 2$ additional observations on each unknown.

Step (3): If $k=1$ then go to Step (4). Suppose $k>1$. The current array $\boldsymbol{D}_{r}$ is of size $m / 2 \times n$, where $n=2 q+m+(m-1)(r-1)$. Each pair of columns, $i$ and $n+1-i$, still contains all $m$ different unknown specimens, for $i=1,2, \ldots, n / 2$. Let $k_{0}=(k-1) / 2$. Suppose

$$
1 \leqslant i_{1}<i_{2}<\cdots<i_{k_{0}-1}<i_{k_{0}} \leqslant n / 2 .
$$

For each $j=i_{1}, \ldots, i_{k_{0}}$, replace columns $\boldsymbol{c}_{j}$ and $\boldsymbol{c}_{n+1-j}$ by a block of 3 columns, $\boldsymbol{c}_{j}|\boldsymbol{s}| \boldsymbol{c}_{j}$ and $\boldsymbol{c}_{n+1-j}|\boldsymbol{s}| \boldsymbol{c}_{n+1-j}$, respectively. At the end of this step each unknown specimen occurs exactly $t$ times in the rows of the modified array $\boldsymbol{D}_{r}$.

Step (4): Add a column of $S$ 's before the first column of the current array $\boldsymbol{D}_{r}$ and a single $S$ following the element in the last row and last column. This yields a (nonrectangular) array with $m$ rows. Concatenate the rows of this array to produce a single row of standards and unknowns. This leads to a balanced measurement design meeting all requirements except that it has $b_{0}-2$ fewer measurements on standards than what is required and also has $b_{0}-2$ fewer empty batches. If $b_{0}=2$ then we are done. If $b_{0}>2$, then we introduce $b_{0}-2$ additional standards in the design sequence by placing them adjacent to already occurring standards. Many different choices will be available here but any one of them will work. This yields the final design.

Note: The choice of the columns $i_{1}<i_{2}<\cdots<i_{k_{0}}$ in Step (3) determines the batch sizes in the final design. Let $2 t=(k-1) u+v$ where $u$ and $v$ are nonnegative integers and $|u-v|$ is as small as possible. Choose $i_{j}=j u-(j-1)$ for $j=1, \ldots, k_{0}$. This choice leads to $(k-1) m / 2$ batches of size $u$ and $m / 2$ batches of size $v$, thus producing nearly equal batch sizes. Other choices of the columns are also possible leading to a different definition of batches.

The following three examples are given to illustrate the algorithm.

Example 4.2. Suppose $m=5, r=0, q=3, t=5, b=12$, and $b_{0}=2$. A balanced measurement design with these parameters can be constructed according to Case (A) of the algorithm above. Note that the value of $k$ here is 2 and also that $t=5=u k+v$ with $u=2$ and $v=1$. Hence construct the following array $\boldsymbol{D}$ first.

$$
\boldsymbol{D}=\begin{array}{lll}
U_{1} & U_{1} & \\
U_{1} & U_{1} & U_{1} \\
U_{2} & U_{2} & \\
U_{2} & U_{2} & U_{2} \\
U_{3} & U_{3} & \\
U_{3} & U_{3} & U_{3} \\
U_{4} & U_{4} & \\
U_{4} & U_{4} & U_{3} \\
U_{5} & U_{5} & \\
U_{5} & U_{5} & U_{3}
\end{array}
$$


After adding the standards we get

$$
\begin{aligned}
& \begin{array}{lll}
S U_{1} & U_{1}
\end{array} \\
& \begin{array}{llll}
S & U_{1} & U_{1} & U_{1}
\end{array} \\
& \begin{array}{lll}
S & U_{2} & U_{2}
\end{array} \\
& \begin{array}{llll}
S & U_{2} & U_{2} & U_{2}
\end{array} \\
& \boldsymbol{D}=\begin{array}{llll}
S & U_{3} & U_{3} \\
S & U_{3} & U_{3} & U_{3}
\end{array} \\
& \begin{array}{lll}
\mathrm{S}_{4} & U_{4}
\end{array} \\
& \begin{array}{lllll}
S & U_{4} & U_{4} & U_{3}
\end{array} \\
& \begin{array}{lll}
S & U_{5} & U_{5}
\end{array} \\
& \begin{array}{lllll}
S & U_{5} & U_{5} & U_{3} & S
\end{array}
\end{aligned}
$$

Concatenating the rows of the above array gives us the required measurement design, viz.,

$$
\begin{array}{llllllllllllllllllllll}
S & U_{1} & U_{1} & S & U_{1} & U_{1} & U_{1} & S & U_{2} & U_{2} & S & U_{2} & U_{2} & U_{2} & S & U_{3} & U_{3} & S & U_{3} & U_{3} & U_{3} & S \\
& & & & & U_{4} & U_{4} & S & U_{4} & U_{4} & U_{4} & S & U_{5} & U_{5} & S & U_{5} & U_{5} & U_{5} & S
\end{array}
$$

Example 4.3. Suppose $m=5, r=3, q=1, t=9, b=14$ and $b_{0}=4$. A balanced measurement design satisfying these parameters can be obtained from Case (B) of the algorithm described above.

Step (1): From Example 4.1,

$$
\begin{aligned}
& \begin{array}{lllllll}
U_{5} & U_{1} & U_{4} & \vdots & U_{4} & U_{1} & U_{5}
\end{array} \\
& \begin{array}{lllllll}
U_{1} & U_{2} & U_{5} & \vdots & U_{5} & U_{2} & U_{1}
\end{array} \\
& \boldsymbol{A}_{1} \vdots \boldsymbol{A}_{2}=U_{4} U_{5} U_{3} \vdots U_{3} U_{5} U_{4} \\
& \begin{array}{lllllll}
U_{2} & U_{3} & U_{1} & \vdots & U_{1} & U_{3} & U_{2}
\end{array} \\
& \begin{array}{llllllll}
U_{3} & U_{4} & U_{2} & \vdots & U_{2} & U_{4} & U_{3}
\end{array}
\end{aligned}
$$

Since $r=3$,

$$
\begin{aligned}
& \begin{array}{lllllll}
U_{5} & U_{1} & U_{4} & U_{1} & U_{5} & U_{1} & U_{4}
\end{array} \\
& \begin{array}{llllllll}
U_{1} & U_{2} & U_{5} & U_{2} & U_{1} & U_{2} & U_{5}
\end{array} \\
& \boldsymbol{D}_{r}=\boldsymbol{A}_{1} \circ \boldsymbol{A}_{2} \circ \boldsymbol{A}_{1}=U_{4} U_{5} U_{3} U_{5} U_{4} U_{5} U_{3} \\
& \begin{array}{lllllll}
U_{2} & U_{3} & U_{1} & U_{3} & U_{2} & U_{3} & U_{1}
\end{array} \\
& \begin{array}{llllllll}
U_{3} & U_{4} & U_{2} & U_{4} & U_{3} & U_{4} & U_{2}
\end{array}
\end{aligned}
$$

Step (2): Since $q=1$, repeating the last column of the current $\boldsymbol{D}_{r}$ gives

$$
\begin{aligned}
& \begin{array}{llllllll}
U_{5} & U_{1} & U_{4} & U_{1} & U_{5} & U_{1} & U_{4} & U_{4}
\end{array} \\
& \begin{array}{lllllllll}
U_{1} & U_{2} & U_{5} & U_{2} & U_{1} & U_{2} & U_{5} & U_{5}
\end{array}
\end{aligned}
$$

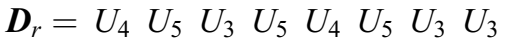

$$
\begin{aligned}
& \begin{array}{lllllllll}
U_{2} & U_{3} & U_{1} & U_{3} & U_{2} & U_{3} & U_{1} & U_{1}
\end{array} \\
& \begin{array}{llllllllll}
U_{3} & U_{4} & U_{2} & U_{4} & U_{3} & U_{4} & U_{2} & U_{2}
\end{array}
\end{aligned}
$$


Step (3): $b-b_{0}=10=k m$, with $k=2$. Since $t=9$, we have $u=5$ and $v=4$. The 5 th column
$U_{5}$
$U_{1}$
$U_{4}$
$U_{2}$
$U_{3}$

is replaced by the block of three columns

$$
\begin{array}{lll}
U_{5} & S & U_{5} \\
U_{1} & S & U_{1} \\
U_{4} & S & U_{4} \\
U_{2} & S & U_{2} \\
U_{3} & S & U_{3}
\end{array}
$$

and we obtain the array

$$
\begin{array}{llllllllll}
U_{5} & U_{1} & U_{4} & U_{1} & U_{5} & S & U_{5} & U_{1} & U_{4} & U_{4} \\
U_{1} & U_{2} & U_{5} & U_{2} & U_{1} & S & U_{1} & U_{2} & U_{5} & U_{5} \\
U_{4} & U_{5} & U_{3} & U_{5} & U_{4} & S & U_{4} & U_{5} & U_{3} & U_{3} \\
U_{2} & U_{3} & U_{1} & U_{3} & U_{2} & S & U_{2} & U_{3} & U_{1} & U_{1} \\
U_{3} & U_{4} & U_{2} & U_{4} & U_{3} & S & U_{3} & U_{4} & U_{2} & U_{2}
\end{array}
$$

Step (4): Add a column of $S$ 's before the first column and a single $S$ at the end of the last row and get the following nonrectangular array.

$$
\begin{aligned}
& \begin{array}{lllllllllllllll}
S & U_{5} & U_{1} & U_{4} & U_{1} & U_{5} & S & U_{5} & U_{1} & U_{4} & U_{4}
\end{array} \\
& \begin{array}{llllllllllll}
S & U_{1} & U_{2} & U_{5} & U_{2} & U_{1} & S & U_{1} & U_{2} & U_{5} & U_{5}
\end{array} \\
& \begin{array}{llllllllllllll}
S & U_{4} & U_{5} & U_{3} & U_{5} & U_{4} & S & U_{4} & U_{5} & U_{3} & U_{3}
\end{array} \\
& \begin{array}{llllllllllllll}
S & U_{2} & U_{3} & U_{1} & U_{3} & U_{2} & S & U_{2} & U_{3} & U_{1} & U_{1}
\end{array} \\
& \begin{array}{lllllllllllllll}
S & U_{3} & U_{4} & U_{2} & U_{4} & U_{3} & S & U_{3} & U_{4} & U_{2} & U_{2} & S
\end{array}
\end{aligned}
$$

Concatenating the rows of this array produces the following design:

$$
\begin{array}{cccccccccccccccccccc}
S & U_{5} & U_{1} & U_{4} & U_{1} & U_{5} & S & U_{5} & U_{1} & U_{4} & U_{4} & S & U_{1} & U_{2} & U_{5} & U_{2} & U_{1} & S & U_{1} & U_{2} \\
U_{5} & U_{5} & S & U_{4} & U_{5} & U_{3} & U_{5} & U_{4} & S & U_{4} & U_{5} & U_{3} & U_{3} & S & U_{2} & U_{3} & U_{1} & U_{3} & U_{2} & S \\
U_{2} & U_{3} & U_{1} & U_{1} & S & U_{3} & U_{4} & U_{2} & U_{4} & U_{3} & S & U_{3} & U_{4} & U_{2} & U_{2} & S & & & &
\end{array}
$$

Since $b_{0}=4, b_{0}-2=2$, and we add two additional standards each adjacent to a previously occurring standard. The final design is given below.

$$
\begin{array}{cccccccccccccccccccc}
S & U_{5} & U_{1} & U_{4} & U_{1} & U_{5} & S & U_{5} & U_{1} & U_{4} & U_{4} & S & S & U_{1} & U_{2} & U_{5} & U_{2} & U_{1} & S & U_{1} \\
U_{2} & U_{5} & U_{5} & S & U_{4} & U_{5} & U_{3} & U_{5} & U_{4} & S & U_{4} & U_{5} & U_{3} & U_{3} & S & S & U_{2} & U_{3} & U_{1} & U_{3} \\
U_{2} & S & U_{2} & U_{3} & U_{1} & U_{1} & S & U_{3} & U_{4} & U_{2} & U_{4} & U_{3} & S & U_{3} & U_{4} & U_{2} & U_{2} & S & &
\end{array}
$$

Example 4.4. Suppose $m=6, r=3, q=0, t=10, b=17$ and $b_{0}=2$. A balanced measurement design satisfying these parameters can be obtained from Case $(C)$ of the algorithm as follows. 
Step (1): From Example 4.1,

$$
\begin{aligned}
& \begin{array}{lllllllllllllllllllll}
U_{6} & U_{1} & U_{5} & U_{2} & U_{4} & U_{3} & \vdots & U_{3} & U_{4} & U_{2} & U_{5} & U_{1} & U_{6}
\end{array} \\
& \boldsymbol{A}_{1} \vdots \boldsymbol{A}_{2}=U_{1} U_{2} U_{6} U_{3} U_{5} U_{4} \vdots U_{4} U_{5} U_{3} U_{6} U_{2} U_{1}
\end{aligned}
$$

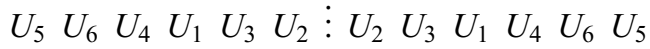

Since $r=3$,

$$
\boldsymbol{D}_{r}=\boldsymbol{A}_{1} \circ \boldsymbol{A}_{2} \circ \boldsymbol{A}_{1}=\begin{array}{lllllllllllllllll}
U_{6} & U_{1} & U_{5} & U_{2} & U_{4} & U_{3} & U_{4} & U_{2} & U_{5} & U_{1} & U_{6} & U_{1} & U_{5} & U_{2} & U_{4} & U_{3} \\
& U_{1} & U_{2} & U_{6} & U_{3} & U_{5} & U_{4} & U_{5} & U_{3} & U_{6} & U_{2} & U_{1} & U_{2} & U_{6} & U_{3} & U_{5} & U_{4} \\
& U_{5} & U_{6} & U_{4} & U_{1} & U_{3} & U_{2} & U_{3} & U_{1} & U_{4} & U_{6} & U_{5} & U_{6} & U_{4} & U_{1} & U_{3} & U_{2}
\end{array}
$$

Step (2): $q=0$. Keep the current array. We have $n_{1}=16$.

Step (3): $b-b_{0}=15=k m / 2$, with $k=5$. Since $t=10$, we get $2 t=(k-1) 4+4$ so $u=4$ and $v=4$. Each row of $\boldsymbol{D}_{r}$ is split at the 4th, 7th, 10th and 13th elements and this gives

$$
\begin{array}{cccccccccccccccccccc}
U_{6} & U_{1} & U_{5} & U_{2} & S & U_{2} & U_{4} & U_{3} & U_{4} & S & U_{4} & U_{2} & U_{5} & U_{1} & S & U_{1} & U_{6} & U_{1} & U_{5} & S \\
U_{5} & U_{2} & U_{4} & U_{3} & U_{1} & U_{2} & U_{6} & U_{3} & S & U_{3} & U_{5} & U_{4} & U_{5} & S & U_{5} & U_{3} & U_{6} & U_{2} & S & U_{2} \\
U_{1} & U_{2} & U_{6} & S & U_{6} & U_{3} & U_{5} & U_{4} & U_{5} & U_{6} & U_{4} & U_{1} & S & U_{1} & U_{3} & U_{2} & U_{3} & S & U_{3} & U_{1} \\
U_{4} & U_{6} & S & U_{6} & U_{5} & U_{6} & U_{4} & S & U_{4} & U_{1} & U_{3} & U_{2} & & & & & & &
\end{array}
$$

Step (4): Add a column of $S$ 's before the first column and a single $S$ at the end of the last row to get the following nonrectangular array:

$$
\begin{array}{llllllllllllllllllll}
S & U_{6} & U_{1} & U_{5} & U_{2} & S & U_{2} & U_{4} & U_{3} & U_{4} & S & U_{4} & U_{2} & U_{5} & U_{1} & S & U_{1} & U_{6} & U_{1} & U_{5} \\
S & U_{5} & U_{2} & U_{4} & U_{3} & S & U_{1} & U_{2} & U_{6} & U_{3} & S & U_{3} & U_{5} & U_{4} & U_{5} & S & U_{5} & U_{3} & U_{6} & U_{2} \\
S & U_{2} & U_{1} & U_{2} & U_{6} & S & U_{6} & U_{3} & U_{5} & U_{4} & S & U_{5} & U_{6} & U_{4} & U_{1} & S & U_{1} & U_{3} & U_{2} & U_{3} \\
S & U_{3} & U_{1} & U_{4} & U_{6} & S & U_{6} & U_{5} & U_{6} & U_{4} & S & U_{4} & U_{1} & U_{3} & U_{2} & S & & & &
\end{array}
$$

Concatenating the rows of the above array we get the design sequence shown below.

$$
\begin{array}{llllllllllllllllllll}
S & U_{6} & U_{1} & U_{5} & U_{2} & S & U_{2} & U_{4} & U_{3} & U_{4} & S & U_{4} & U_{2} & U_{5} & U_{1} & S & U_{1} & U_{6} & U_{1} & U_{5} \\
S & U_{5} & U_{2} & U_{4} & U_{3} & S & U_{1} & U_{2} & U_{6} & U_{3} & S & U_{3} & U_{5} & U_{4} & U_{5} & S & U_{5} & U_{3} & U_{6} & U_{2} \\
S & U_{2} & U_{1} & U_{2} & U_{6} & S & U_{6} & U_{3} & U_{5} & U_{4} & S & U_{5} & U_{6} & U_{4} & U_{1} & S & U_{1} & U_{3} & U_{2} & U_{3} \\
S & U_{3} & U_{1} & U_{4} & U_{6} & S & U_{6} & U_{5} & U_{6} & U_{4} & S & U_{4} & U_{1} & U_{3} & U_{2} & S & & &
\end{array}
$$

Since $b_{0}=2$, no more standards need to be added.

\section{Concluding remarks}

In this paper we have carried out a detailed analysis of exact optimum balanced measurement designs for three or more unknowns, assuming an $\mathrm{AR}(1)$ error structure. For the case of less than three unknowns the reader is referred to Taylor (1989). Although the measurement design problem presented here is equivalent to the treatment-control design problem considered by others, the optimum designs derived in this paper are not obtainable from existing results. 
It is customary to prove results that guarantee that certain families of designs exhibiting some balance properties are optimal among all competing designs. However, designs exhibiting such balance may not always exist for user specified values of $N$, the total number of runs, and $m$, the number of unknowns. Therefore, we have focused on the actual construction of balanced optimum measurement designs with the user having complete freedom to choose the values for $m$ and $N$. Special cases of optimal balanced designs may turn out to be optimum among all possible designs, but we have not pursued this problem here. We do, however, point out how lower bounds for the trace criterion may be obtained by using the well known symmetrization argument. The calculations required for obtaining optimum balanced designs and lower bounds for the trace criterion are easily implemented on a computer.

In principle the approach can be extended to higher-order autoregressive error structures but an analytical treatment of exact optimum designs seems unfeasible. In these cases it may be necessary to consider approximately optimum designs. In practical applications low order $\mathrm{AR}(1)$ error models are often useful as approximations to the actual serial correlation structure. Thus the designs presented in this paper can provide useful information in these cases as well.

\section{Appendix}

Proof of Theorem 3.1. Suppose $-1<\phi<0$. The equality in (2.7) implies that $b-$ $b_{0}=m x$ for some $x$ where $x$ must be a positive integer when $(m-1) r$ is even and a positive odd multiple of $1 / 2$ when $(m-1) r$ is odd. We substitute $b-b_{0}=m x$ in the expression for the trace in (3.1) and

$$
\operatorname{Trace}\left(\boldsymbol{C}^{-1}\right)=\frac{m-1}{t(1-\phi)^{2}+\frac{2 x \phi}{m}+m r \phi}+\frac{1}{t(1-\phi)^{2}+\frac{2 x \phi}{m}-\frac{m t^{2}(1-\phi)^{3}}{2+(N-2)(1-\phi)}} .
$$

We minimize this expression with respect to allowable values of $x_{0}$ and $r$ for each fixed $t$. We consider several cases.

(1) Suppose $m$ is odd and $b-2 \geqslant m$. For a fixed $r,($ A.1) is minimized by a small $x$. Due to the constraints given in $(2.7)-(2.9)$ and since $(m-1) r$ is even in this case for every allowable $r, x$ must be a positive integer in the interval

$$
\max \left\{\frac{1}{m}, 1-\frac{(m-1) r}{2}\right\} \leqslant x \leqslant \min \left\{t-\frac{(m-1) r}{2}, \frac{b-2}{m}\right\} .
$$

Hence $x$ must be chosen to be 1 . We substitute $x=1$ in the expression for the trace in (A.1) and minimize the resulting expression with respect to $r$. Again, since $\phi$ is negative, we must choose the smallest allowable value for $r$. Since $r$ must belong to the interval given by

$$
\max \left\{0, \frac{2(m-b+2)}{m(m-1)}\right\} \leqslant r \leqslant \frac{2(m t-1)}{m(m-1)}
$$

and since the lower bound for $r$ in this case is zero, we take $r=0$. Therefore, we have $r=0$ and $b_{0}=b-m$. 
(2) Suppose $m$ is an even number greater than $2 t$ and $b-2 \geqslant m$. Here we consider two subcases $-r$ odd and $r$ even.

If $r$ is odd, then $(m-1) r$ is odd, so $x$ must be an odd positive multiple of $1 / 2$. However, since $m$ is greater than $2 t$, it can be easily verified that there are no such values for $x$ and hence a balanced design does not exist in this case.

If $r$ is even, then $(m-1) r$ is even, so $x$ must be a positive integer. As in case (1) $x$ must be chosen to be 1 . Substituting $x=1$ in (A.1) and minimizing with respect to $r$ yields $r=0$. So the solution in this case is given by $r=0$ and $b_{0}=b-m$.

(3) Suppose $m$ is an even number less than or equal to $2 t$ and $b-2 \geqslant m$. Again we consider two subcases $-r$ odd and $r$ even.

If $r$ is odd, then $(m-1) r$ is odd, so $x$ must be an odd positive multiple of $1 / 2$. The smallest allowable value for $x$ in this case is $x=1 / 2$. Substituting $x=1 / 2$ in (A.1) and minimizing with respect to $r$ gives $r=1$. Therefore the solution in this subcase is $r=1$ and $b_{0}=b-m / 2$.

If $r$ is even, then $(m-1) r$ is even, so $x$ must be a positive integer. As in case (2) $x$ must be chosen to be 1 . Substituting $x=1$ in (A.1) and minimizing with respect to $r$ yields $r=0$. So the solution in this subcase is given by $r=0$ and $b_{0}=b-m$.

Putting the two subcases together we find that the optimum design is the better of the two designs obtained in the two subcases.

(4) Here we have $1 / 2 \leqslant(b-2) / m<1$ and $m$ is an even integer less than or equal to $2 t$. If $r$ is an even integer we find that the set of allowable values for $x$ is empty. So $r$ must be chosen to be odd. For a fixed odd positive $r$ the trace is minimized by taking $x=1 / 2$. Substituting this in (A.1) and minimizing with respect to $r$ gives $r=1$. The solution in this cases is then $r=1$ and $b_{0}=b-m / 2$.

(5) Clearly no balanced design exists in the remaining cases because the set of allowable values for $(x, r)$ is empty for these cases.

Hence we have proved Theorem 3.1.

Proof of Theorem 3.2. As in the case of Theorem 3.1, we have $b-b_{0}=m x$ where $x$ is an integer when $(m-1) r$ is even and it is an odd positive multiple of $1 / 2$ when $(m-1) r$ is odd. Substituting $m x$ for $b-b_{0}$ in (3.1) gives us the expression in (A.1). We now consider each of the cases.

(1) Here we have $m$ even and $1 / 2 \leqslant(b-2) / m \leqslant t$. We consider four subcases.

Suppose $r$ is an odd integer in the interval

$$
\max \left\{0, \frac{2(m-b+2)}{m(m-1)}\right\} \leqslant r \leqslant \frac{2(m t-b+2)}{m(m-1)} .
$$

Then $x$ must be an odd positive multiple of $1 / 2$. The expression in (A.1) is minimized with respect to $x$ by choosing $x$ to be as large as possible within its range of allowable values. It is easy to verify that $x$ cannot exceed $(b-2) / m$ under this case, so we choose $x$ to be the largest odd positive multiple of $1 / 2$ that is less than or equal to $(b-2) / m$. Call this value $x_{0}$. We substitute $x=x_{0}$ in (A.1) and minimize the resulting expression with respect to $r$. Since $\phi$ is positive, we must pick $r$ to be as large as possible. 
So $r$ is chosen to be equal to $r_{0}$, the largest odd positive integer in the interval in (A.2).

Suppose $r$ is an even integer in the interval in (A.2). Then $x$ must be a positive integer. The expression in (A.1) is minimized with respect to $x$ by choosing $x$ to be as large as possible within its range of allowable values. Under this subcase, we must then pick $x$ to be the largest integer, say $x_{0}$, less than or equal to $(b-2) / m$. We substitute $x=x_{0}$ in (A.1) and minimize the resulting expression with respect to $r$. Again, we must pick $r$ as large as possible. So we chose $r=r_{0}$, the largest even nonnegative integer in the interval in (A.2).

Suppose $r$ is an odd integer in the interval

$$
\frac{2(m t-b+2)}{m(m-1)}<r \leqslant \frac{2(m t-1)}{m(m-1)} .
$$

Then $x$ is an odd positive multiple of $1 / 2$ within its range of allowable values. It is easily verified that we must chose $x$ to be equal to $x_{0}$ where $x_{0}$ is given by $x_{0}=t-$ $(m-1) r / 2$. We then substitute $x=x_{0}$ in (A.1) and minimize the resulting expression with respect to $r$. By examining the first derivative of this expression with respect to $r$ it can be verified that it is increasing for values of $r$ in the interval in (A.3). So we choose $r$ to be the smallest odd integer, say $r_{0}$, in the interval given in (A.3). This leads to a design for which $r=r_{0}$ and $b_{0}=b-m t+m(m-1) r_{0} / 2$.

Suppose $r$ is an even integer in the interval in (A.3). Then $x$ must be a positive integer within its range of allowable values. Again this leads to the choice $x=x_{0}$ where $x_{0}$ is $t-(m-1) r / 2$. Substituting this value for $x$ in (A.1) and minimizing with respect to $r$ leads to the choice $r=r_{0}$ where $r_{0}$ is the smallest even integer in the interval in (A.3).

By examining the best designs obtained for the above four subcases we choose the overall best design when $m$ is an even integer with $1 / 2 \leqslant(b-2) / m \leqslant t$. This proves part (1) of Theorem 3.2.

Suppose $m$ is odd and $1 \leqslant(b-2) / m \leqslant t$. Here we consider two subcases. For values of $r$ in the interval in (A.2) $x$ must be the largest positive integer, say $x_{0}$, less than or equal to $(b-2) / m$. Then $r$ must be the largest integer, say $r_{0}$, in the interval in (A.2). For values of $r$ in the interval in (A.3) $x$ must be equal to $x_{0}$ were $x_{0}$ is the value $t-(m-1) r / 2$. Then $r$ must be the smallest integer in the interval in (A.3) because the trace can be shown to be a nondecreasing function of $r$ for values of $r$ in the interval in (A.3).

Putting the two subcases together we obtain the result in part (2) of Theorem 3.2.

For case (3) of Theorem 3.2, we have $(b-2) / m>t$. Here $x$ must be chosen as $x_{0}=t-(m-1) r / 2$. We substitute this expression for $x$ in (A.1) and note that the resulting expression is a nondecreasing function of $r$ for all allowable values of $r$. So we pick the smallest possible value of $r$, i.e., we choose $r=0$. Hence $x=t$ and $b_{0}=b-m t$.

It is easy to verify that a balanced design does not exist in the remaining cases. This concludes the proof of Theorem 3.2. 


\section{References}

Berenblut, R.E., Webb, G.I., 1974. Experimental design in the presence of autocorrelated errors. Biometrika $61,427$.

Cheng, C.S., 1983. Construction of optimal balanced incomplete block designs for correlated observations. Ann. Statist. 11, 204-209.

Cheng, C.S., 1988. A note on the optimality of semibalanced arrays. In: Dodge, Y., Fedorov, V.V., Wynn, H.P. (Eds.), Optimal Design and Analysis of Experiments. North-Holland, Amsterdam, pp. 115-122.

Cutler, D.R., 1993. Efficient block designs for comparing test treatments to a control when the errors are correlated. J. Statist. Plann. Inference 36, 107-125.

Fedorov, V.V., 1972. Theory of Optimal Experiments. Academic Press, New York.

Hedayat, A.S., Jacroux, M., Majumdar, D., 1988. Optimal designs for comparing test treatments with controls. Statist. Sci. 3, 462-476.

Ipinyomi, R.A., 1986. Equineighboured experimental designs. Aust. J. Statist. 28, 79-88.

Kiefer, J., 1960. Optimum experimental designs. J. Roy. Statist. Soc. Ser. B 21, 272-319.

Kiefer, J., 1975. Construction and optimality of generalized youden designs. In: Srivastava, J.N. (Ed.), A Survey of Statistical Design and Linear Models, pp. 333-353.

Kiefer, J., Wynn, H.P., 1981. Optimal balanced block and latin square designs for correlated observations. Ann. Statist. 9, 737-757.

Kiefer, J., Wynn, H.P., 1983. Autocorrelation-robust design of experiments. In: Leonard, T., Wu, C.F. (Eds.), Scientific Inference, Data Analysis and Robustness, p. 279.

Kiefer, J., Wynn, H.P., 1984. Optimum and minimax exact treatment designs for one dimensionl autoregressive error processes. Ann. Statist. 12, 414-450.

Kunert, J., 1985a. Optimal experimental design when the errors are assumed to be correlated. Statist. Decisions. Suppl. 2, 287-298.

Kunert, J., 1985b. Optimal repeated meaurements designs for correlated observations and analysis by weighted least squares. Biometrika 72, 375-389.

Kunert, J., 1987. Neighbour balanced block designs for correlated errors. Biometrika 74, 717-724.

Kunert, J., Martin, R.J., 1987. On the optimality of finite Williams II(a) designs. Ann. Statist. 15, 1604-1628.

Martin, R.J., 1982. Some aspects of experimental design and analysis when errors are correlated. Biometrika 69, 597-612.

Martin, R.J., Eccleston, J.A., 1991. Optimal incomplete block designs for general dependence structures. J. Statist. Plann. Inference 28, 67-81.

Majumdar, D., 1988. Optimal repeated meaurements designs for comparing test treatments with control. Commun. Statist. — Theory Meth. 17, 3687-3703.

Pepper, M.P.G., 1973. A calibration of instruments with non-random errors. Technometrics 15, 587-599.

Pigeon, G., Raghavarao, D., 1987. Crossover designs for comparing treatments with a control. Biometrika 74, 321-328.

Russell, K.G., Eccleston, J.A., 1987a. The construction of optimal balanced incomplete block designs when adjacent observations are correlated. Aust. J. Statist. 29, 84-90.

Russell, K.G., Eccleston, J.A., 1987b. The construction of optimal balanced incomplete block designs when observations within a block are correlated. Aust. J. Statist. 29, 293-302.

Siddiqui, M.M., 1958. On the inversion of the sample covariance matrix in a stationary autogressive process. Ann. Math. Statist. 29, 585-588.

Taylor, C.H., 1989. Optimal measurement designs when errors are correlated. Ph.D. Dissertation, Colorado State University.

Williams, E.J., 1949. Experimental designs balanced for the estimation of residual effects and treatments. Aust. J. Sci. Res. 2, 149-168.

Williams, E.R., 1985. A criterion for the construction of optimal neighbour designs. J. Roy. Statist. Soc. Ser. B 47, 489-497.

Williams, E.R., 1986. A neighbour model for field experiments. Biometrika 73, 279-287. 